\title{
AR Guide for Food Allergic Consumers
}

\author{
Karthika. $\mathrm{S}^{\mathrm{a}, 1}$, Varsha. $\mathrm{G}^{\mathrm{b}}$ and Deepika. $\mathrm{R}^{\mathrm{c}}$ \\ ${ }^{a, b}$ Student, , Dept of CSE, Sri Sairam Engineering College, Chennai \\ ${ }^{c}$ Assistant Professor, Dept of CSE, Sri Sairam Engineering College, Chennai
}

\begin{abstract}
Augmented reality is a technology where real life environment is enhanced by incorporating digital or virtual elements like images, graphics, 3D objects into it. Augmented reality is a growing and trending technology which can make interactions easier when incorporated into normal applications. The aim of this project is to create an augmented reality food guide for food allergic consumers to aid them in choosing allergen free foods. A person who wants to order food online or in restaurants doesn't know whether it would be suitable for him to eat or not. Especially if they are allergic to certain foods, they have to be preventive and cautious in choosing the type of food they eat. This AR guide will aid them in choosing the suitable food for them in an augmented environment where a consumer can view food in three dimension view along with the necessary information about the food such as the key ingredients and the presence of any allergens that will be helpful in choosing the allergen free food.
\end{abstract}

Keywords: Augmented reality, 3d modelling, food labelling.

\section{Introduction}

Augmented Reality (AR) is a recently emerging technology that enhances the real life environment by incorporating computer generated two or three dimensional elements such as texts, images, graphics, etc. It offers enhanced level of interaction with the environment by employing virtual information to it. An augmented reality application (AR) provides flexible and user interactive learning and visualizing environment to users. AR is mostly used in education for demonstrating concepts that require realistic visual objects. However it can also be applied in various fields such as entertainment, healthcare and food industry. There are many systems for food ordering online. But these systems do not provide any means to help the consumers who are allergic to certain foods. Also, sometimes even non allergic consumers may find it difficult to order food online if it is something out of their regular pick. In such case, they might have to do searches online to learn about the kind of food they are ordering. There is no system to visualize food the way it is in the real environment along with the necessary health information that can aid those with allergic reactions to certain foods before they order them. However augmented reality can be a perfect solution for this. Using three dimensional models of the food along with the vital information about the food can be

'Karthika S, Student, , Dept of CSE, Sri Sairam Engineering College, Chennai;

E-mail:karthibhatia06@gmail.com 
very useful to both allergic and non-allergic consumers in choosing their foods according to their preferences. Using target images such as markers or by detecting flat surfaces augmented reality application can scan images through the camera of a smart phone and can generate 3D models of the food that can help both the allergic and nonallergic consumers in ordering the food of their preference without any confusion. This paper aims to discuss the development and implementation of an augmented reality (AR) application aiding both allergic and non-allergic consumers in choosing the food of their preference. Section 2 discusses the related works; Section 3 discusses the proposed system methodology; Section 4 discusses the design modules; Section 5 discusses the results and this paper concludes at Section 6.

\section{Related work}

Meghan E. Rollo et al. (2017) did a detailed study on an AR app called servAR to check to what extent it can help in estimating carb choices of the food. The app showed carb choices on a real dish virtually using an iPad Mini which included nine foods such as pasta, green beans, rice, corn, broccoli, potatoes, cauliflower, kidney beans and carrots. The study reported that the ServAR app showed improved carb-choice accuracy and great potential as a tool to support the estimation of carb choices.[7] AR Breakfast (Calle-Bustos et al., 2017) is an augmented reality app that is developed for supporting therapeutic education for diabetic patients. It helps the patients learn about the carb choices of foods they eat. This app places virtual food on a marker along with the carb information so the patients can learn the carb choices of the food they eat. The results showed that the diabetic patients earlier unaware of the carb choices learnt about them after using the app.

\section{Proposed system methodology}

A sample application with a simple user interface is developed to test the performance practically. The user interface allows the user to choose a dish from a cuisine. This AR guide will have $3 \mathrm{~d}$ food models along with the necessary information about the food which will be projected virtually in real time using target detection and $3 \mathrm{~d}$ augmentation techniques. A common target will be set and the $3 \mathrm{~d}$ food models along with the information will be projected on that target which will help the consumers in choosing the food to eat.

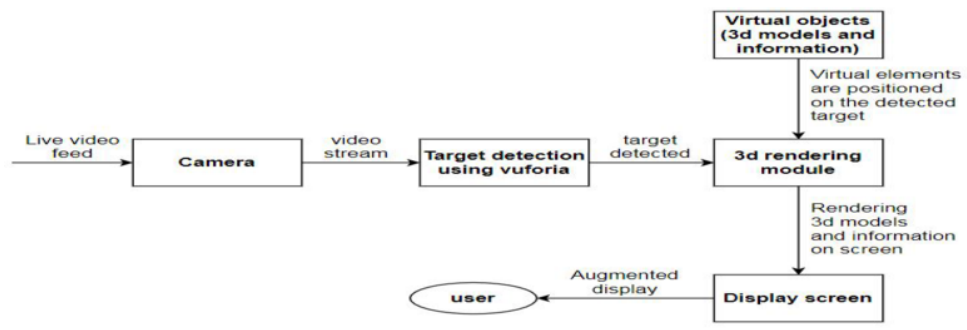

Figure 1. Block diagram representing the process 


\section{Design module}

\subsection{D model constructions}

Reference images are collected. Basic shapes are scaled, sculpted and adjusted in blender to create a rough model. The model is rendered by applying suitable modifiers to create a textured model. The materials and textures are applied to the model in unity.

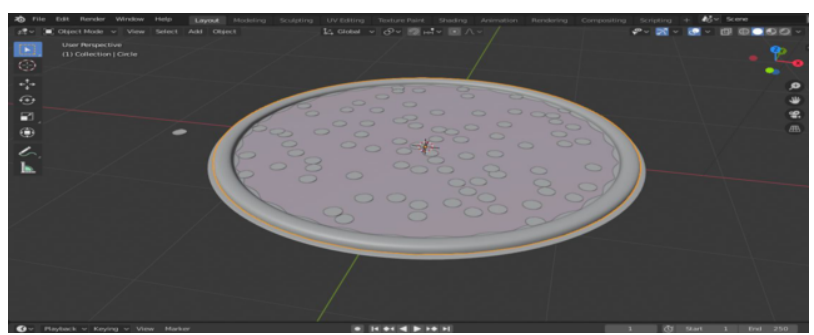

Figure 2. Rendering a model in blender

\subsection{Target detection and $3 d$ model augmentation}

\subsubsection{Target detection}

Vuforia is one of the famous sdk used for target detection and it has many tools required for creating an AR experience.Vuforia detects the target at run time in the live camera feed by comparing the features of the target with that of the features extracted in the predefined target image. Vuforia rates the target to be used from 0 to 5 stars. The higher the rating, the greater and more accurate the detection.

\subsection{2 $3 d$ model augmentation}

After target detection, $3 \mathrm{~d}$ models are mounted on the target image so as to display them on the target whenever the target image is shown on the camera. Augmentable target image that has distinct features is chosen and set as a target. The target is adjusted in the scene as per the position of the camera coordinates. $3 \mathrm{~d}$ model is positioned on the target image and programmed to be activated on button click. The process is repeated for all the models in different scenes. 


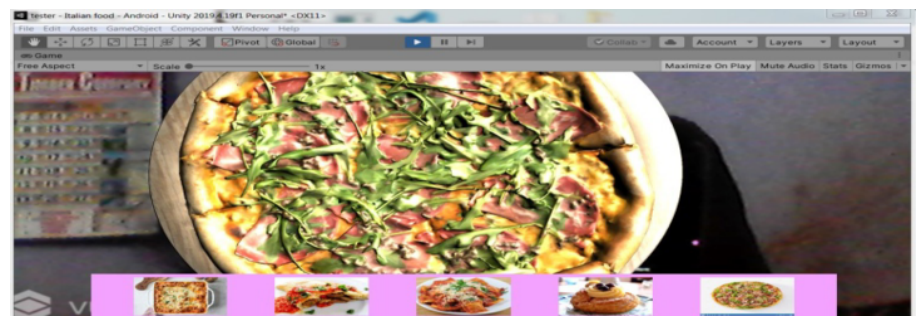

Figure 3. 3d model augmentation "Pizza" (https://skfb.ly/6RqYo) by Rigsters

\subsection{Information augmentation}

Information about the food featured are collected from various sources and classified as allergens, key ingredients, directions and recipe and consolidated to facilitate the data feed. The information is displayed in four different columns that the consumer can see along with the $3 \mathrm{~d}$ display of the food.

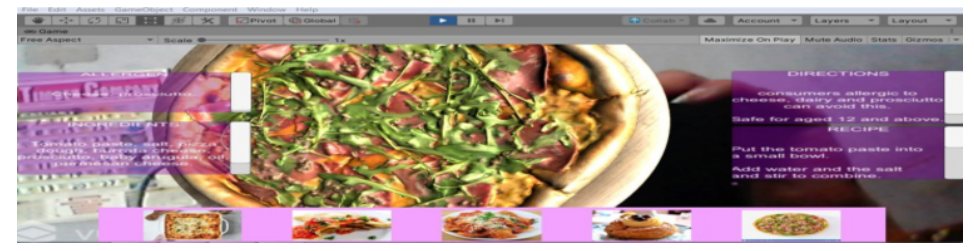

Figure 4. Augmented display containing $3 \mathrm{~d}$ model of food and its information.

\subsection{User interface}

User interface acts as a bridge between the user and the application. User interface is an important factor in application development process. A good flexible user interface lets the user to interact with the application with ease. A good application will have user friendly interface that will allow the users to interact with the application without any prior knowledge about the application. In this, user interface will have home page where the list of cuisines will be displayed in buttons. A click on the button will lead to another scene where there will be dishes of the respective cuisine featured. The application will show the $3 \mathrm{~d}$ model of the dish clicked along with its information on the screen when the target is shown to the camera.

\section{Results and future enhancements}

The above approach can be applied in restaurants and hotels to ensure the safety and wellbeing of their customers. This approach not only helps food allergic consumers but also non allergic consumers in choosing the kind of food they eat. This approach only acts as an aid guide for the consumers but it can be enhanced in future by detecting a 
nearby restaurant that has the foods featured in this application with the help of gps services and providing a means to order that particular food online without having to look for the places elsewhere.

\section{Conclusion}

Here the food allergic consumer can check if the food is suitable for them to eat or not by checking the information about the food displayed on the screen. The information will let the consumer know if the food contains any common allergen that most people find harmful. If the consumer is allergic to any other allergen apart from the listed allergens, they can check if it is present in the ingredients used in the dish. If the consumer is very sensitive to food and that they don't want to take any risks, by ordering food elsewhere, they can make their own allergen free food themselves by following up the recipe given in the application. This application can aid both allergic and non allergic consumers in choosing the food they want to eat. It is satisfactory and practical as long as it is used for authentic foods.

\section{References}

[1] Veronica Rossano, Rosa Lanzilotti, Antonio Cazzolla, Teresa Roselli. Augmented Reality to Support Geometry Learning. IEEE. Volume 8, June 2020.

[2] Rubaiya Hafiz, Jahidul Hasan, Md. Azizul Hakim. Enhancement of Human Anatomy and Nervous System Learning using Mobile Augmented Reality Application. International Journal of Engineering and Advanced Technology (IJEAT) ISSN: 2249 - 8958. Volume-9 Issue-3, February, 2020.

[3] Xiuquan Qiao, Pei Ren, Schahram Dustdar, Ling Liu, Huadong Ma, Junliang Chen. Web AR: A promising future for mobile augmented reality- State of the art, challenges and insights. IEEE Trans. In AR, vol.107, no.4, April 2019.

[4] Eg Su Goh, Mohd Shahrizal Sunar, Ajune Wanis Ismail. 3D Object Manipulation Techniques in Handheld Mobile Augmented Reality Interface: A Review. VOLUME 7, 2019.

[5] Jibin Yin, Chengyao Fu, Xiangliang Zhang, Tao Liu. Precise Target Selection Techniques in Handheld Augmented Reality Interfaces. VOLUME 7, 2019.

[6] Swarna Priya RM, Aarthy S L, Gunavathi C, Venkatesh P, Srinivas Koppu. 3d reconstruction of a scene from multiple $2 \mathrm{~d}$ images. International Journal of Civil Engineering and Technology (IJCIET). Volume 8, Issue 12, December 2017.

[7] Megan E. Rollo, Tamara Bucher, Shamus P. Smith \& Clare E. Collins. ServAR: An augmented reality tool to guide the serving of food. International Journal of Behavioural Nutrition and Physical Activity. Volume 14, Article number: 65 (2017).

[8] Anuroop Katiyar , Karan Kalra and Chetan Garg. Marker Based Augmented Reality. Advances in Computer Science and Information Technology (ACSIT). Print ISSN: 2393-9907; Online ISSN: $2393-$ 9915; Volume 2, Number 5; April-June, 2015 pp. 441-445.

[9] Isidro Navarro,Antonio Galindo,David Fonseca. Augmented reality uses in educational research projects: The "Falcones Project", a case study applying technology in the Humanities framework at high school level. First International Conference on Technological Ecosystems for Enhancing Multiculturality (TEEM'13), 2013.

[10] Christina A. Roberto, MS, Peter D. Larsen, MPhil, Henry Agnew, BA, Jenny Baik, BA, and Kelly D. Brownwell,PhD. Evaluating the impact of menu labelling on food choices and intake. American journal of public health,2010.

[11] Ambeth Kumar.V.D et,al .Enhancement in Footprint Image using Diverse Filtering Technique.Procedia Engineering journal, Volume 8, No.12, 1072-1080, 2012 . [doi:10.1016/j.proeng.2012.01.965] 